EPJ Web of Conferences 53, 08002 (2013)

DOI: $10.1051 /$ epjconf/20135308002

(C) Owned by the authors, published by EDP Sciences, 2013

\title{
Multimuon detector for quasi horizontal EAS
}

\author{
P. Spillantini \\ University and INFN, Firenze, Italy
}

\begin{abstract}
Quasi horizontal showers produced by charged CR and gammas are constituted exclusively by muons, the other components being absorbed in the thick layer of the atmosphere they must cross to reach a detection system on the Earth surface. Their transverse dimensions are increased by the distance they must travel as well by the terrestrial magnetic field which extends muon cloud and separates positive muons from negative ones. The extended wide transverse dimensions allow increasing the rate of detection of EAS produced by high energy CR by means of a single detector which samples the muon density in the shower. This concept has been already applied in the NEVOD-DECOR experimental complex at MEPhI in Moscow that could detect local muon density (LMD) of showers produced by very high energy CR up to more than $10^{18} \mathrm{eV}$. By adding to the LMD the measurement of the local energy density (LED) the determination of the primary energy $\mathrm{E}_{0}$ can be substantially improved. A few embodiments of such a device can be envisaged and one of them is discussed. The substantial rate increase of the area explored by a multimuon detection system measuring LMD and LED allows to conceive an array constituted by a relatively small number of such stations for measuring UHECR up to $10^{12} \mathrm{eV}$ and higher.
\end{abstract}

\section{INTRODUCTION}

The muon content of EAS produced by UHECRs increases at the increasing of the zenith angle. Quasi horizontal showers are exclusively constituted by muons, the other components being absorbed in the thick layer of the atmosphere they must cross for reaching the Earth surface.

The transverse dimensions of the muon cloud are increased by the distance they must travel to reach ground as well by the terrestrial magnetic field which tends to extend their width and also separate positive muons from negative ones (Fig. 1).

These facts increase the rate of UHECR EAS that impinge on a fixed ground position: muons and muon bundles can be detected at large distances from the core of the shower on a very wide region (several $10^{6} \mathrm{~m}^{2}$ ), quite sufficient for obtaining statistically significant results up to primary particle energies of $10^{18} \mathrm{eV}$ and higher. Muon bundles are for one order of magnitude nearer to the core of the shower than single muon, and their muons are for one order of magnitude more energetic than the single muon spectrum. Therefore the measurement of local muon density $\mathrm{D}_{\mu}$ is highly sensitive to the most energetic hadrons produced in the forward region of hadron interactions. It carries important information where the uncertainties in model predictions are maximal and there are not accelerator data.

\section{MEASUREMENT OF THE MUON DENSITY IN UHECR EAS}

The increase of rate is substantial. It depends from the area of the muon detector which measures $\mathrm{D}_{\mu}$. Requiring $\mathrm{D}_{\mu}>7$ on $\mathrm{a} \approx 100 \mathrm{~m}^{2}$ detector, at $\mathrm{E}_{\mathrm{o}}=10^{18} \mathrm{eV}$ the collection area of one station measuring EAS arriving from $80^{\circ}$ zenith angle is of the order of $0.6 \times 2.5 \mathrm{~km}^{2}$ perpendicularly to the shower axis, i.e. $3 \times 2.5 \mathrm{~km}^{2}$ on ground (see right plot in Fig. 2).

This is an Open Access article distributed under the terms of the Creative Commons Attribution License 2.0, which permits unrestricted use, distribution, and reproduction in any medium, provided the original work is properly cited. 


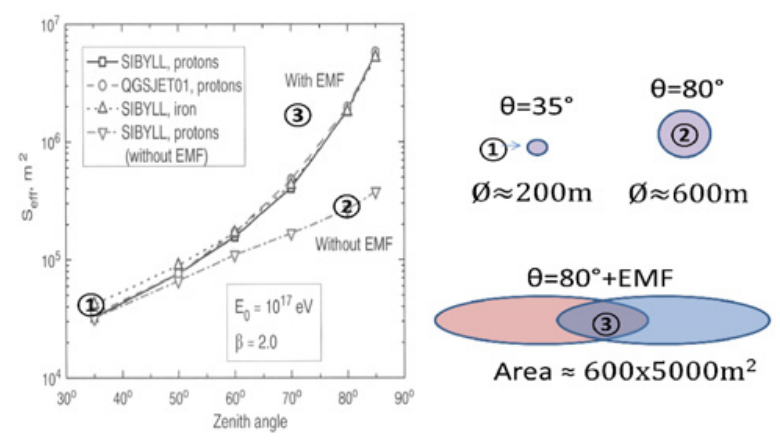

Figure 1. Effective area of EAS collection in Local Muon Density Spectra technique. Lower curve: calculation with 'switched-off' Earth magnetic field (EMF).
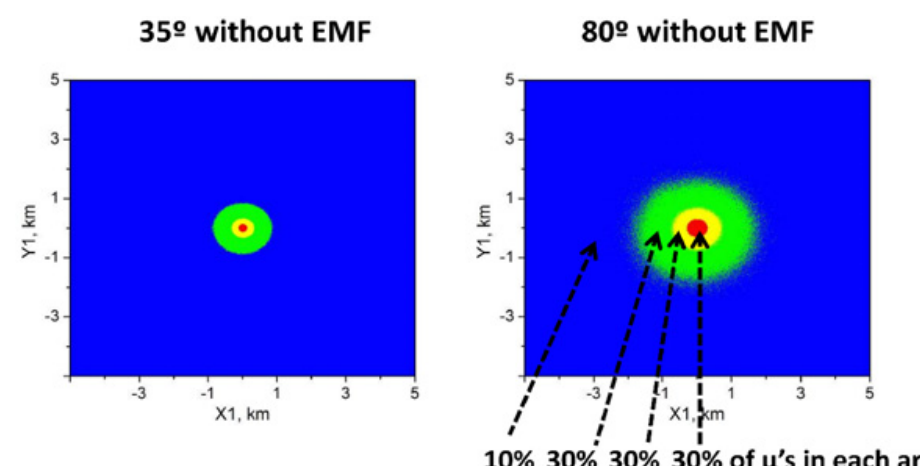

$10 \% 30 \% 30 \% 30 \%$ of $\mu$ 's in each area

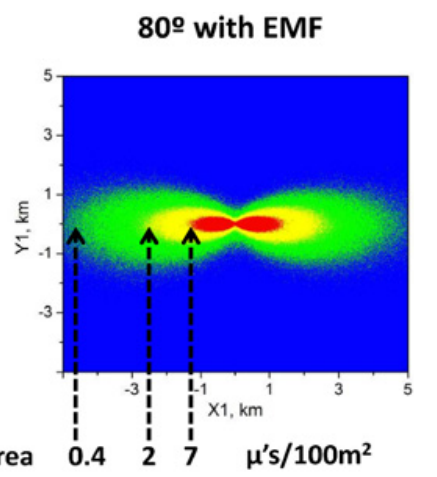

protons, $\mathrm{E}_{0}=10^{18} \mathrm{eV}$

(EMF=Earth Magnetic Field @ latitude 50)

Figure 2. Effective area of EAS collection in Local Muon Density Spectra technique. The content of muons is $30 \%$ in each area. In third figure the averaged density of muons is indicated for $\mathrm{E}_{\mathrm{o}}=10^{18} \mathrm{eV}$ energy of the primary CR.

The easier parameter to be measured is the local areal density $\mathrm{D}_{\mu}$ of the impinging muon bundles. This is the method used by the DECOR instrument [1] deployed around the Cherenkov water calorimeter NEVOD [2] at MEPhI in Moscow, which could detect local muon densities of showers produced by UHECR up to more than $10^{18} \mathrm{eV}$.

Due to the vertical orientation of DECOR planes and the presence of coordinate detector modules faced in two orthogonal azimuth directions, the azimuth acceptance of NEVOD-DECOR complex is nearly uniform $\left(25-40 \mathrm{~m}^{2}\right)$ and allows collection of nearly horizontal multimuon events arriving from all azimuth angles. The data collected during several years allowed distinguishing different production models of shower in the atmosphere up to the very high energy $10^{18} \mathrm{eV}$ [3] by one relatively simple measurement station.

\section{MEASUREMENT OF THE MUON ENERGY DENSITY IN UHECR EAS}

The initial energy $\mathrm{E}_{\mathrm{o}}$ of the UHECR originating the shower is determined with a large error $\left(\sigma\left(\log \mathrm{E}_{\mathrm{o}}\right) \approx\right.$ 0.4) on the basis of a statistical treatment [4].

By adding to the measurement of the $\mathrm{D}_{\mu}$ parameter the measurement on the same position on ground of the averaged energy $\left\langle\mathrm{E}_{\mu}\right\rangle$ of the EAS muon cloud the error on $\mathrm{E}_{\mathrm{o}}$ can be substantially reduced allowing 


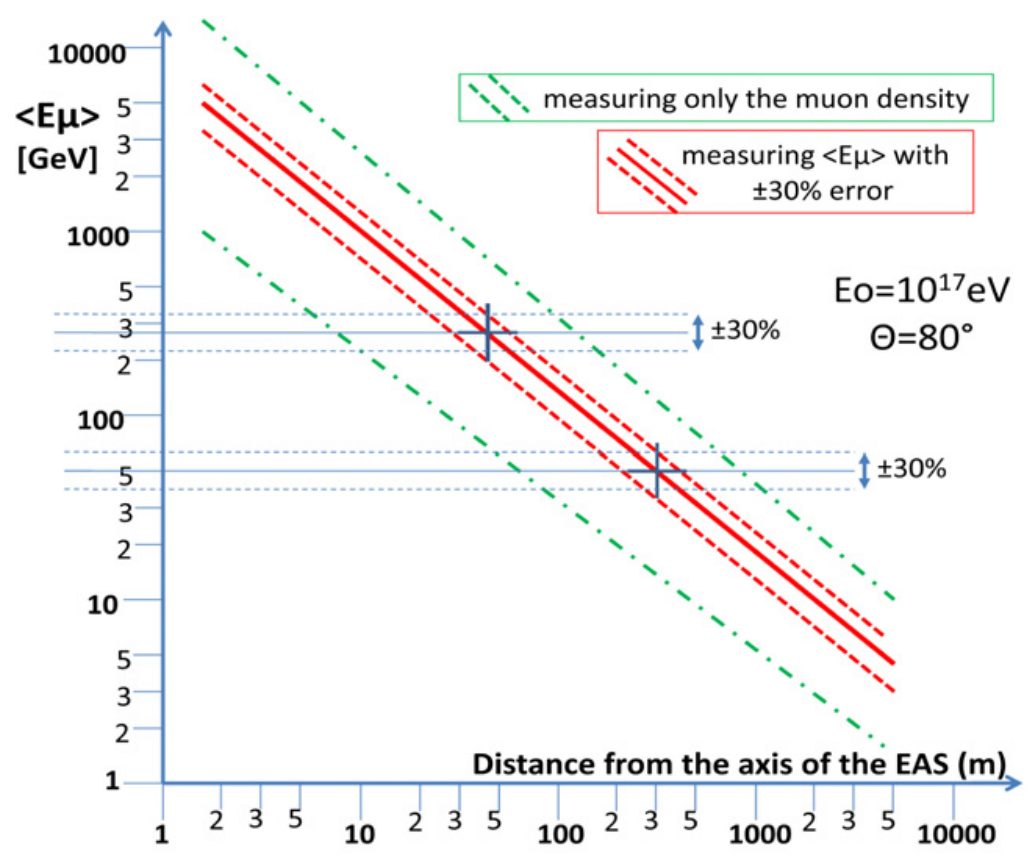

Figure 3. Correlation between the averaged muon averaged energy $\left\langle\mathrm{E}_{\mu}\right\rangle$ and the distance from the core of the shower for $\theta=80^{\circ}$ and $\mathrm{E}_{\mathrm{o}}=10^{18} \mathrm{eV}$. The two dash-dotted lines correspond to the wide range of variability of the correlation. By measuring $\left\langle\mathrm{E}_{\mu}\right\rangle$ with an error $( \pm 30 \%$, dashed lines in the figure) the correlation is improved.

to determine the flux and the energy spectrum of the UHECR. This is represented in Figure 3, where in double log scale the local muon averaged energy $\left\langle\mathrm{E}_{\mu}\right\rangle$ is reported vs the distance from the axis of the shower for $\mathrm{E}_{\mathrm{o}}=10^{18} \mathrm{eV}^{1}$.

In the figure the two dash-dotted lines indicate the wide range of variability of this correlation. By measuring $\left\langle\mathrm{E}_{\mu}\right\rangle$ with an error (e.g. 30\%, dashed lines in the figure) the correlation with the distance from the EAS core is greatly improved. The $\mathrm{D}_{\mu}$ measurement (to be conceived as a third axis perpendicular to the plane of the figure) can determine $\mathrm{E}_{\mathrm{o}}$ with a relatively small error.

\section{EMBODIMENTS OF THE APPARATUS FOR MEASURING $E_{\mu}$ IN THE MULTIMUON DETECTION SYSTEM}

\subsection{Measurement of muon momentum by a magnetized iron spectrometer}

A possible embodiment of an apparatus for measuring $\mathrm{E}_{\mu}$ can be based on one magnetized iron system surrounded by tracking detectors for measuring the muon directions (and also $\mathrm{D}_{\mu}$ ); the energy of each muon is measured by tracking it before and after the magnetized iron. The measurement of the energy of each muon not only measures the energy density $\mathrm{E}_{\mu}$ but also the local muon energy spectrum, further improving the determination of the position of the core of the shower and of the energy $E_{0}$ of the primary particle.

The thickness of the iron to be crossed by the muons depends from the precision we require to reach in the muon momentum measurement. In order to obtain $<20 \%$ error the thickness of the magnetized iron to be crossed must be in the range 3-4 $\mathrm{m}$. The iron cannot be one block because of the need to

\footnotetext{
${ }^{1}$ The plot in Figure 3 only slightly depends from $\mathrm{E}_{\mathrm{o}}$.
} 


\section{EPJ Web of Conferences}
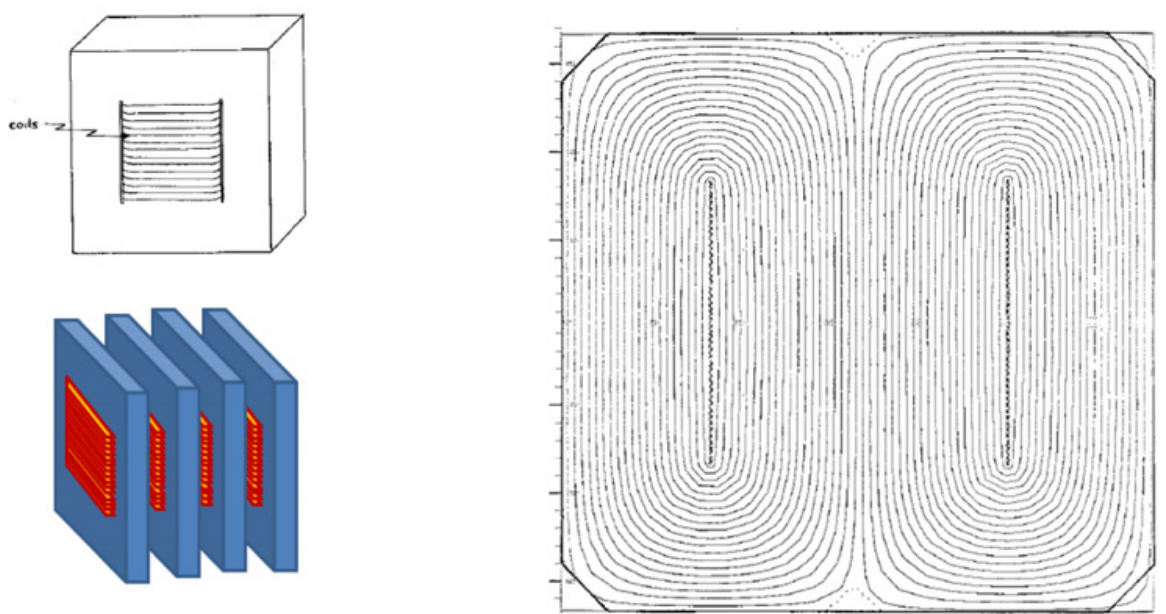

Figure 4. Scheme of the magnetized iron magnet to be inserted in the muon density measurement system for measuring the muon momentum.

3 gaps $\approx 0.2 \mathrm{~m}$ for the tracker and the coils encoumbrance

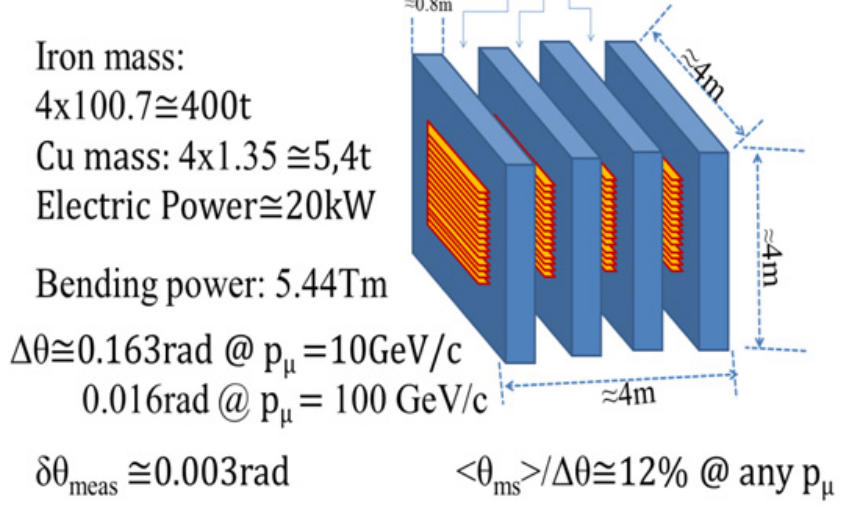

Figure 5. Dimensions and main parameters of a possible magnetized iron spectrometer.

follow each muon to be measured, and also to identify and measure those that are stopped by range inside the magnet. A possible scheme for the magnetized iron magnet is reported in Figure 4.

The magnetized iron block can be similar to that proposed thirty years ago for the project of the "Jura neutrino experiment" [5]. It can be subdivided in 4 layers, each magnetized as indicated in Figure 4. The main parameters reported in Figure 5 are evaluated for the dimensions reported in the figure.

The required electric power is somewhat low, about $5 \mathrm{~kW}$ per iron layer, what can be provided also in desert areas by a relatively small solar panel plant. A system of three detectors for the pattern recognition of the muons of the bundle must be interleaved between the iron layers.

The layout of the station for the measurement of $\mathrm{D}_{\mu}$ and $\mathrm{E}_{\mu}$ and its main components are sketched in Figure 6 . The muon momentum is measured by its angular deflection by the multimuon tracker system external to the magnet. 


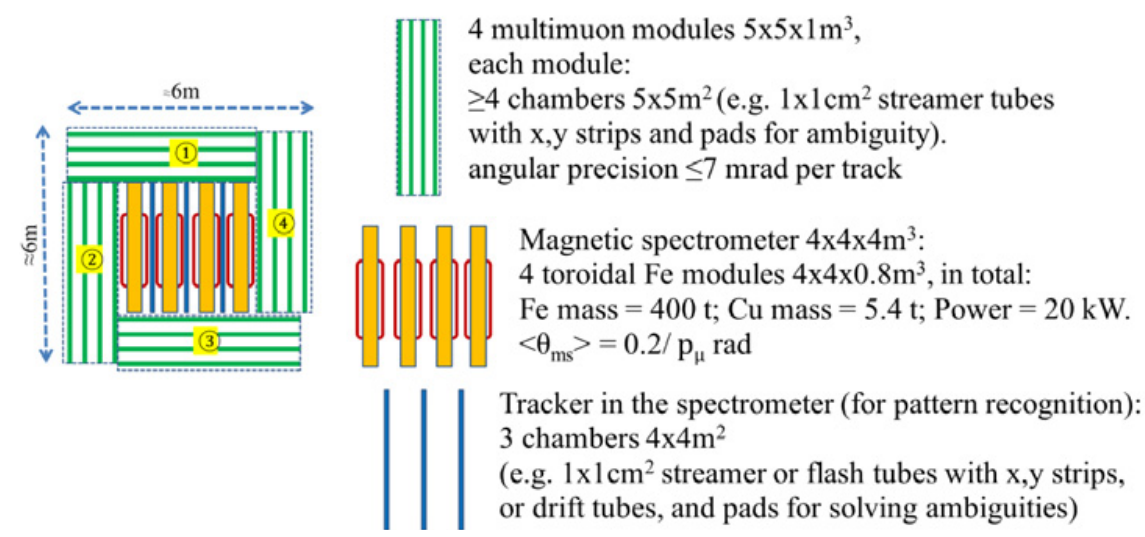

Figure 6. Layout and main components of a multimuon measurement station.

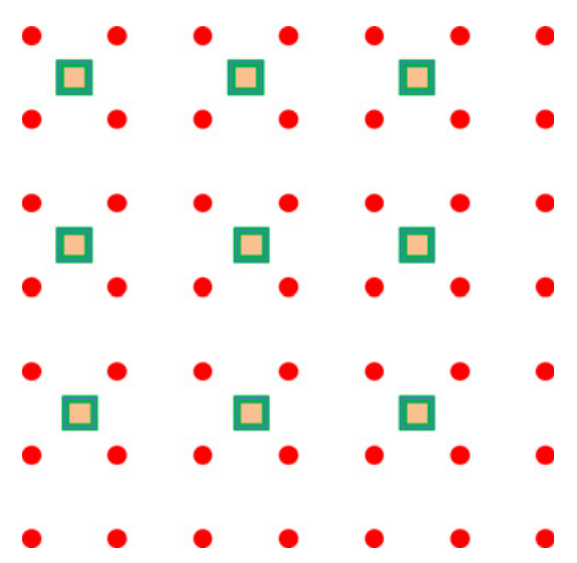

Figure 7. A system of multimuon measurement stations (pitch $3 \mathrm{~km}$ ) over imposed to the AUGER surface array (pitch $1.5 \mathrm{~km}$ ).

\subsection{Other possible systems for measuring the muon energy in the multimuon detection station}

Other embodiments of the idea are possible, such as the energy measurement of the most energetic muons by large area TRDs based on the detection of the very hard X-rays produced in dense radiators at large angles respect to the direction of the crossing muon [6], or the measurement of the density of clusters along the muon track. Their needed large geometrical dimensions require a dedicated R\&D in order to be discussed in some detail and evaluated their convenience.

\section{SYSTEM OF MULTIMUON DETECTORS FOR QUASI HORIZONTAL UHECR SHOWERS}

It is interesting and worthwhile considering how the increase of rate of detection of UHECR can be used for extending the collection area of giant UHECR detection systems.

In fact the substantial increase of the area explored by one multimuon detection station measuring both $\mathrm{D}_{\mu}$ and $\mathrm{E}_{\mu}$ allows to conceive an array constituted by a relatively small number of such stations for measuring the UHECR spectrum up to $10^{19} \mathrm{eV}$ and higher. Such an array would be somewhat less dense than the Auger array for covering comparable area and $\mathrm{E}_{\mathrm{o}}$ range (Fig. 7). 


\section{EPJ Web of Conferences}

An Auger-type array could be complemented with multimuon detection stations measuring both $\mathrm{D}_{\mu}$ and $\mathrm{E}_{\mu}$; the Auger-type array would help to determine the position of the core of the shower allowing a technical simplification of the multimuon detection stations and the minimizing their number.

The final ambitious goal of such an approach could be the realization of an UHE neutrino observatory. It should initially be optimized for working in the primary energy $\mathrm{E}_{\mathrm{o}}$ range between $10^{18}$ and $10^{19} \mathrm{eV}$ where the so called cosmogenic neutrinos should be found and measured.

The author wishes to thank Prof. A.Petrukhin and the team of his NEVOD-DECOR laboratory for the advices and support in developing the ideas reported in this work.

\section{References}

[1] N.D. Barbashina et al., Instrum.Exp.Tech. 43, 743-746 (2000)

[2] V.M. Aynutdinov et al., Astrophys. Space Sci. 258, 105-116 (1997)

[3] A.A. Petrukhin, "Cosmic Ray above the knee; experimental results and their interpretation" Proc. 32 ${ }^{\text {nd }}$ ICRC, Beijing, China 1, 50-53 (2011). Available in http://icrc2011.ihep.ac. $\mathrm{cn} /$ new.htm

[4] A.G. Bogdanov et al., Phys. Tom. Nucl. 73, 1852-1869 (2010)

[5] A. Grant et al., "Search for neutrino oscillations in the SPS wide-band beam", CERN/SPSC/81-14, SPSC/P158 (1981)

[6] M.L. Cherry and G.L. Case, "Compton scattered transition radiation from very high energy particles”, Astroparticle Physics 18, 629-635 (2003) 